\title{
Downregulation of hsa_circ_0000285 serves as a prognostic biomarker for bladder cancer and is involved in cisplatin resistance
}

\author{
B. J. CHI ${ }^{1,2}$, D. M. ZHAO ${ }^{1}$, L. $\mathrm{LIU}^{2}$, X. Z. YIN ${ }^{2}$, F. F. WANG ${ }^{2}$, S. BI ${ }^{1}$, S. L. GUI' ${ }^{1}$, S. B. ZHOU ${ }^{2,3}$, W. B. QIN ${ }^{4}$, D. M. WU ${ }^{5}$, S. Q. WANG ${ }^{2, *}$ \\ ${ }^{1}$ Department of Urology, The First Affiliated Hospital of Jiamusi University, Jiamusi, China; ${ }^{2}$ Basic Medical College, Jiamusi University, Jiamusi, \\ China; ${ }^{3}$ School of Life Sciences, Institute of Biomedical and Environmental Science and Technology, University of Bedfordshire, Luton, United \\ Kingdom; ${ }^{4}$ Department of Urology, The Second Affiliated Hospital of Jiamusi University, Jiamusi, China; ${ }^{5}$ Material College, Jiamusi University, \\ Jiamusi, China \\ *Correspondence: jimusisqwang@sohu.com
}

Received March 18, 2018 / Accepted July 16, 2018

\begin{abstract}
Bladder cancer remains a very challenging disease to treat with the high rates of recurrence and progression associated with current therapies. Although the association between bladder cancer pathology and circRNAs remains undetermined, circRNAs signatures may be useful as prognostic and predictive factors and clinical tools for assessing disease state, treatment response and outcome. This study investigates if these circRNAs can be used as biomarkers for bladder cancer diagnosis and predicting treatment response. Herein, qPCR measured the expression of hsa_circRNA_100783, hsa_circ_0000285 and hsa_circRNA_100782 in bladder cancer tissues. It was established that sa_circ_0000285, but not hsa_circRNA_100782 and hsa_circRNA_10078, are significantly reduced in bladder cancer tissues and serum compared to adjacent tissues and healthy controls. Moreover, hsa_circ_0000285 expression was lower in cisplatin-resistant bladder cancer patients than in those who were cisplatin-sensitive. Here, hsa_circ_0000285 was associated with tumor size $(\mathrm{p}<0.001)$, differentiation $(\mathrm{p}<0.001)$, lymph node metastasis $(p=0.038)$, distant metastasis $(p=0.004)$ and TNM stage $(p=0.013)$. Further analysis showed that hsa_circ_0000285 would be an independent prognostic factor for bladder cancer patient outcome. In conclusion, our study indicates hsa_circ_0000285 may be a novel biomarker for bladder cancer because of its involvement in bladder cancer chemo-sensitivity.
\end{abstract}

Key words: circRNA, bladder cancer, circHIPK3, resistance, chemo-sensitivity

Bladder cancer remains a very challenging disease, with the high rates of recurrence and progression associated with current therapies [1]. Despite significant medical advancement, conventional platinum-based chemotherapy remains first line therapy for advanced urothelial carcinoma [2]. However, cisplatin-resistance to this disease plagues bladder cancer treatment [3]. Until recently, the potential pathway or mechanism of bladder cancer was not determined, but the ability to characterize cancer genomes is now providing insights into the genesis and molecular underpinnings of this disease [4]. Increasing evidence indicates that aberrantly expressed non-coding RNAs, including miRNAs, lncRNA and part of circRNAs, are responsible for cancer initiation and progression; this includes bladder cancer [5]. Deregulated circRNAs can act either as tumor suppressors or oncogenes to control cell proliferation, migration, and metastasis [6]. In addition, circRNAs signatures may be used as prognostic and predictive factors for cancers, thus offering a potential clinical tool for assessing disease state and predicting treatment response and clinical outcome [7]. These results highlight the importance of circRNAs in mediating oncogenic processes [8]. Therefore, further examination and validation of de-regulated circRNAs in bladder cancer should provide insight into the fundamental drivers of this disease.

circHIPK3 is an abundant circRNA produced from the HIPK3 gene and it forms a circle through long intronic complementary repeat elements [9]. Silencing circHIPK3 inhibits HuH-7, HCT-116 and HeLa cells proliferation [9]. However, circHIPK3 expression correlates with progression [10] but functions as a tumor suppressor in bladder cancer by sponging miR-558 [11]. Therefore, circHIPK3's biological role appears specific in different cancer types. Although previous studies have shown circHIPK3 is downregulated in human bladder cancer, it remains undetermined if circHIPK3 can be detected in serum samples and if it is associated with clinical features and chemotherapy response as a non-invasive biomarker for bladder cancer. 
The HIPK3 gene locus formats hsa_circRNA_100783, hsa_circ_0000285 and hsa_circRNA_100782, and their genomic locations are listed in Supplementary Table 1. The association between these circRNAs and bladder cancer pathology remains undetermined, therefore we investigated if these circRNAs could be useful as biomarkers for bladder cancer diagnosis and predicting treatment response.

\section{Patients and methods}

Bladder cancer tissues and serum sample collection. Samples were collected from 146 bladder cancer tissues and 98 adjacent tissues at The First Affiliated Hospital of Jiamusi University. Serum samples from another independent cohort, including 97 healthy people and 197 bladder cancer patients, were also collected from this source. The medical records of bladder cancer patients with clinical TNM staging and survival information were then assembled. Cisplatinresistant bladder cancer patients were defined as those with disease persisting more than 6 weeks and recurrent disease more than 2 months after chemotherapy completion, and cisplatin-sensitive bladder cancer patients were those without these conditions. This project was approved by the Ethic Committee of The First Affiliated Hospital of Jiamusi University.

Cell lines. The normal bladder epithelial cells CCC-HB-2 and 6 bladder cancer cell lines (HTB-9, T24, J82, SW780 and RT4) were obtained from the Chinese Academy of Sciences Cellbank. Cells were grown routinely in RPMI-1640 medium (Invitrogen, CA, USA) supplemented with $10 \%$ fetal bovine serum (Gibco, CA, USA) and cultured in a $37^{\circ} \mathrm{C}$ humidified atmosphere of $5 \% \mathrm{CO}_{2}$.

The cisplatin-resistant RT4 cell line (RT4/DDP) was established in our laboratory. The RT4 cells $\left(1 \times 10^{5} / \mathrm{ml}\right)$ were cultured for $24 \mathrm{~h}$, and then treated with the initial concentration of cisplatin $(0.05 \mu \mathrm{g} / \mathrm{ml})$. The medium containing gefitinib was changed every 2 to 3 days. After initial doses were induced for two weeks, drug dosage was doubled, and each dose was maintained for 2 weeks. The final concentration was increased to $0.5 \mu \mathrm{g} / \mathrm{ml}$.
Quantitative PCR analysis. Trizol reagent (Invitrogen, CA, USA) extracted total RNA from cells. ABScript II cDNA First-Strand Synthesis Kit (cat no. RK20400, ABclonal Biotechnology Co., Ltd, Wuhan, China) was used to reverse transcript cDNA from $500 \mathrm{ng}$ of RNA according to the manufacturer's protocol. The sequence of circRNA results were acquired from the database "circBase". The expression of circRNAs was measured by SsoFast EvaGreen supermix (cat no. 1725201, Bio-Rad Laboratories (Shanghai) Co., Ltd. Shanghai, China) according to manufacturers' instructions. Expression of $\beta$-actin was used as an endogenous control. QPCR was performed at the condition: $95^{\circ} \mathrm{C}$ for $3 \mathrm{~min}$, and 39 circles of $95^{\circ} \mathrm{C}$ for $10 \mathrm{~s}$ and $60^{\circ} \mathrm{C}$ for $30 \mathrm{~s}$. The following primers were used: 5'-TATGTTGGTGGATCCTGTTCGGCA-3'(forward), 5'-TGGTGGGTAGACCAAGACTTGTGA-3' (reverse) for circRNA_000285. 5'-TATGTTGGTGGATCCTGTTCGGCA-3' (forward), 5'-TGGTGGGTAGACCAAGACTTGTGA-3' (reverse) for circRNA_100782. 5'-GCTGCGGCCTGACTCTAAG-3' (forward), 5'-TGTGCTTGATGGTGTCCCT-3' (reverse) for circRNA_100783. 5'-TTGTTACAGGAAGTCCCTTGCC-3' (forward), 5'-ATGCTATCACCTCCCCTGTGTG-3' (reverse) for $\beta$-actin.

Statistical analysis. Data from 3 independent experiments processed in SPSS17.0 statistical software is expressed as mean $\pm \mathrm{SD}$, and overall survival rate estimates were calculated by the Kaplan-Meier method with log-rank test. The clinical association between circRNA_000285 expression and clinical-pathological variables in bladder cancer patients was evaluated by chi-square test and differences between groups was estimated by Student's t-test or one-way ANOVA depending on the conditions.A $\mathrm{p}<0.05$ was statistically significant.

\section{Results}

circRNA_000285 is down-regulated in bladder cancer tissues and cell lines. We performed qPCR to measure the expression of circRNA_000285, circRNA_100782 and circRNA_100783 derived from HIPK3, in bladder cancer
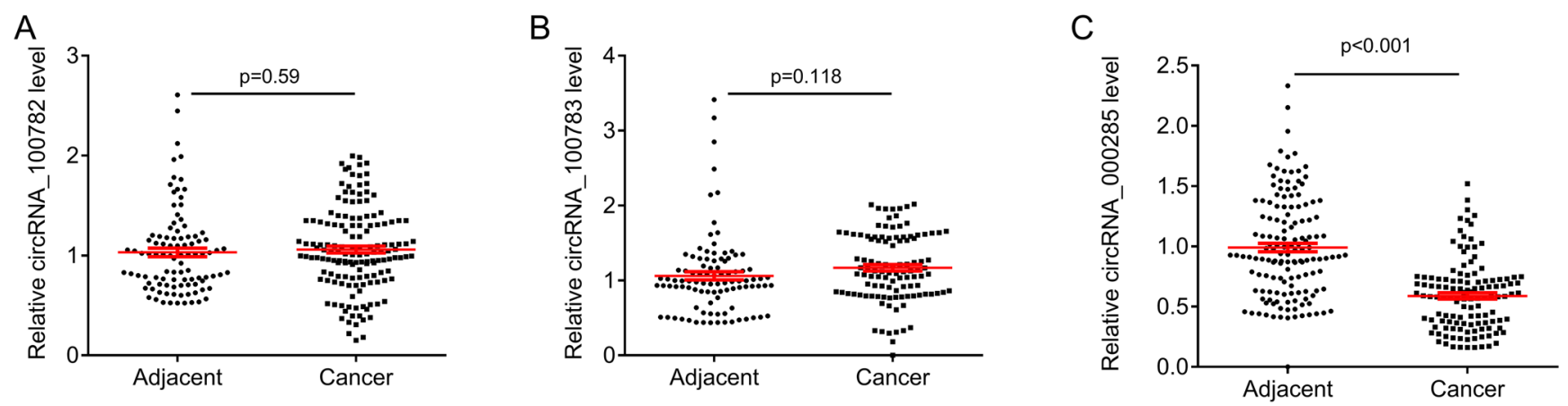

Figure 1. The circRNAs expression in bladder and adjacent tissues. QPCR was performed to measure the expression of hsa_circRNA_100782 (A), hsa_circRNA_100783 (B) and hsa_circ_0000285 (C) in bladder cancer tissues $(\mathbf{n = 1 4 6 )}$ and adjacent tissues (n=98). 
A
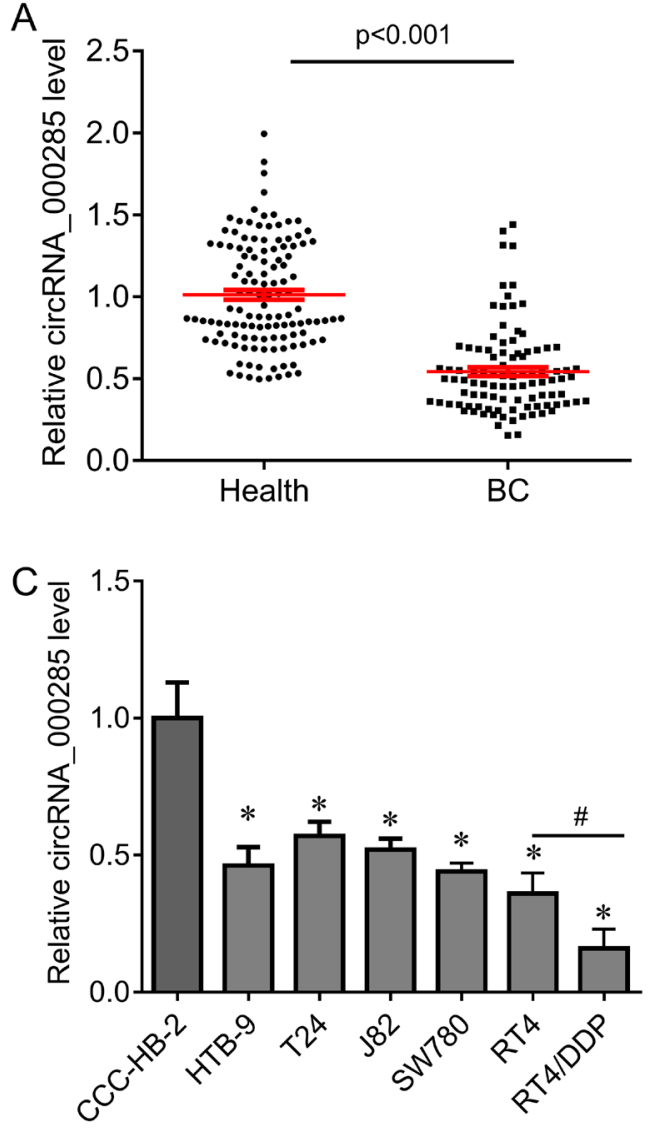

$\mathrm{B}$

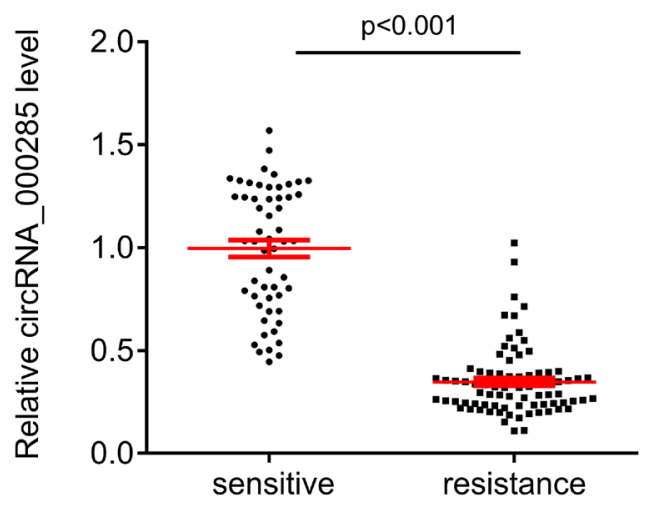

D

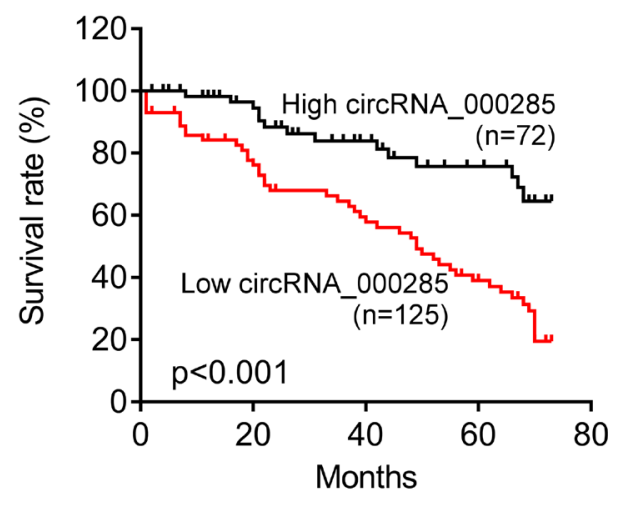

Figure 2. The expression of hsa_circ_0000285 in serum samples. A) QPCR was performed to measure the expression of hsa_circ_0000285 in serum samples from bladder cancer patients $(n=197)$ and health subjects $(n=97)$. B) The expression of hsa_circ_0000285 in serum samples from cisplatinsensitive bladder cancer patients $(n=60)$ and cisplatin-resistant bladder cancer patients $(n=50)$. C) $Q P C R$ was performed to measure the expression of hsa_circ_0000285 in five bladder cancer cells, cisplatin-resistant RT4 cells (RT4/DDP) and normal bladder epithelial cells CCC-HB-2. ${ }^{*}$ p $<0.05$ vs. CCC-HB-2; \#p<0.05. D) The Kaplan-Meier survival cure showed the survival rate in patients with high circRNA_000285 expression ( $\mathrm{n}=72$ ) and those with low circRNA_000285 expression $(\mathbf{n}=125)$.

and adjacent tissues. We found that circRNA_000285 was significantly decreased in bladder cancer tissues compared to adjacent controls (Figure 1C), while the expression of circRNA_100782 and circRNA_100783 was comparable in these tissues (Figure $1 \mathrm{~A}, \mathrm{~B}$ ). However, the host gene HIPK3 mRNA levels had no significant alterations in bladder cancer tissues, serum samples or normal cell lines from adjacent tissues, healthy controls and bladder cancer cell lines (Supplementary Figure 1). We then measured the expression of circRNA_000285 in bladder cancer cell lines and observed that circRNA_000285 was significantly less in these bladder cancer cell lines than in normal epithelial cells (Figure 2C).

circRNA_000285 is downregulated in serum from bladder cancer patients. To investigate whether circRNA_000285 is a useful non-invasive biomarker for bladder cancer, we performed qPCR to detect the expression of circRNA_000285 in an independent cohort, including 197 bladder cancer serum samples and 97 health serum samples. circRNA_000285 was also significantly down-regulated in bladder cancer serum samples compared with to healthy samples (Figure 2A). It was established that circRNA_000285 level was decreased almost three-fold in cisplatin-resistant patients $(n=55)$ compared to the cisplatin-sensitives $(n=50)$ (Figure 2B); and the circRNA_000285 level in cisplatin-resistant RT4 cells was lower than in parental cells (Figure 2C), thus suggesting circRNA_000285 as a biomarker for bladder cancer diagnosis and chemotherapy.

circRNA_000285 is associated with bladder cancer patient clinical features. We next analyzed the association between circRNA_000285 and bladder cancer clinical features. Bladder cancer patients were divided into high circRNA_000285 expression when it was higher than the mean circRNA_000285 levels, and otherwise into the low expression group. We established that circRNA_000285 expression was associated with tumor size $(\mathrm{p}<0.001)$, differentiation $(\mathrm{p}<0.001)$, lymph node metastasis $(\mathrm{p}=0.038)$, distant metastasis $(p=0.004)$ and TNM stage $(p=0.013)$ (Table 1). We then investigated factors that predicate 
bladder cancer patient prognosis by univariate and multivariate analyses. Univariate analysis indicated that the serum circRNA_000285 level $(\mathrm{p}=0.01)$, tumor size $(\mathrm{p}=0.02)$, differentiation $(\mathrm{p}=0.03)$, lymph node metastasis $(\mathrm{p}=0.03)$, distant

Table 1. Clinical association between serum circRNA_000285 levels and clinical--pathological variables of patients with bladder cancer.

\begin{tabular}{|c|c|c|c|}
\hline \multirow[b]{2}{*}{ Variable } & \multicolumn{2}{|c|}{ serum circRNA_000285 } & \multirow{2}{*}{$\begin{array}{l}\chi^{2} \text { test } \\
\text { p-value }\end{array}$} \\
\hline & $\begin{array}{c}\text { Low expression } \\
(\mathrm{n}=125)\end{array}$ & $\begin{array}{c}\text { High expression } \\
(\mathrm{n}=72)\end{array}$ & \\
\hline Age & & & 0.302 \\
\hline$<50$ & 56 & 38 & \\
\hline$\geq 50$ & 69 & 34 & \\
\hline Gender & & & 0.361 \\
\hline Male & 74 & 48 & \\
\hline Female & 51 & 24 & \\
\hline Tumor size & & & $<0.001$ \\
\hline$<3 \mathrm{~cm}$ & 47 & 49 & \\
\hline$\geq 3 \mathrm{~cm}$ & 78 & 23 & \\
\hline Differentiation & & & $<0.001$ \\
\hline High & 20 & 34 & \\
\hline Moderate & 43 & 23 & \\
\hline Low & 62 & 15 & \\
\hline Lymph node metastasis & & & 0.038 \\
\hline N0-1 & 52 & 41 & \\
\hline $\mathrm{N} 2-4$ & 73 & 31 & \\
\hline Distant metastasis & & & 0.004 \\
\hline No & 57 & 48 & \\
\hline Yes & 68 & 24 & \\
\hline TNM stage & & & 0.013 \\
\hline I-II & 50 & 42 & \\
\hline III-IV & 75 & 30 & \\
\hline
\end{tabular}

Table 2. Univariate analysis of prognostic factors of bladder cancer.

\begin{tabular}{lcc}
\hline Variable & Hazard ratio & p-value \\
\hline Age $(\geq 50 /<50)$ & 1.03 & 0.18 \\
Gender (Male/Female) & 1.07 & 0.37 \\
Tumor size $(\geq 3 \mathrm{~cm} /<3 \mathrm{~cm})$ & 2.24 & 0.02 \\
Differentiation (low/high-moderate) & 2.56 & 0.02 \\
Lymph node metastasis (N2-4/N0-1) & 2.76 & 0.03 \\
Distant metastasis (Yes/No) & 3.72 & 0.01 \\
TNM stage (III-IV/I-II) & 3.16 & 0.03 \\
serum circRNA_000285 levels (Low/High) & 3.21 & 0.01 \\
\hline
\end{tabular}

Table 3. Multivariate analysis of independent prognostic factors of bladder cancer.

\begin{tabular}{lcc}
\hline Variable & Hazard ratio & p-value \\
\hline Tumor size & 2.54 & 0.01 \\
Differentiation & 2.26 & 0.04 \\
Lymph node metastasis & 2.73 & 0.02 \\
Distant metastasis & 3.88 & 0.01 \\
TNM stage & 2.93 & 0.01 \\
serum circRNA_000285 levels & 3.03 & 0.02 \\
\hline
\end{tabular}

metastasis $(\mathrm{p}=0.01)$ and TNM stage $(\mathrm{p}=0.03)$ was significantly associated with patient prognosis (Table 2); and multivariate analysis revealed that the serum circRNA_000285 level $(\mathrm{p}=0.02)$, tumor size $(\mathrm{p}=0.01)$, differentiation $(\mathrm{p}=0.04)$, lymph node metastasis $(\mathrm{p}=0.02)$, distant metastasis $(\mathrm{p}=0.01)$ and TNM stage $(\mathrm{p}=0.01)$ were independent factors in predicting bladder cancer patient prognosis.

Finally, we analyzed the relationship between serum circRNA_000285 levels and survival time in bladder cancer patients. The Kaplan-Meier survival cure showed that patients with high circRNA_000285 expression had longer overall survival rate than those with low expression (Figure 2D), thus defining circRNA_000285's critical role in bladder cancer development.

\section{Discussion}

This study established that hsa_circ_0000285 is significantly reduced in bladder cancer tissues and serum compared with adjacent tissues and healthy controls. Its expression is lower in cisplatin-resistant bladder cancer patients than in cisplatin-sensitives and can therefore be an independent prognostic factor for patient outcome. This, however, did not apply to hsa_circRNA_100782 and hsa_circRNA_100783 which were also investigated.

Emerging evidence has revealed the function of circRNAs in cancer and it may potentially serve as a required novel biomarker and therapeutic target for cancer treatment [12]. For example, circ-LDLRAD3 was up-regulated in pancreatic cancer cell lines, tissues and plasma samples and its high expression was significantly associated with patient venous and lymphatic invasion and metastasis [13]. hsa_circ-0000520 is significantly down-regulated in gastric cancer tissues, plasma and cell lines compared to controls, and its expression was negatively associated with TNM stage and plasma linked to CEA expression [14]. Further, Chen $G$ found that the circRNA_100782 was markedly up-regulated in pancreatic ductal adenocarcinoma tissue [15]. Down-regulation of circRNA_100782 inhibited BxPC3 cell proliferation and colony formation by down-regulating the following miR-124 targets; interleukin-6 receptor (IL6R) and signal transducer and activator of transcription 3 (STAT3) [15]. These findings support that circRNAs functions are cancer-specific.

Neo-adjuvant chemotherapy has increased over the last decade, and based on level I evidence, cisplatin-based neo-adjuvant chemotherapy is considered standard care in bladder cancer [16]. While this cisplatin-based chemotherapy before radical cystectomy improves the overall survival of bladder cancer patients, some patients do not benefit from chemotherapy, and the pathological response to neo-adjuvant treatment is a strong predictor of better diseasespecific survival. The identification of reliable biomarkers enabling clinicians to identify patients who could benefit from chemotherapy is a very important clinical task [17]. 
Non-coding RNAs are important mediators of cisplatin-resistance in bladder cancer [18-19]. circTCF25 is up-regulated in bladder cancer and it suppresses $\mathrm{miR}-103 \mathrm{a}-3 \mathrm{p} / \mathrm{miR}-107$, leading to the up-regulation of thirteen targets of cell proliferation, migration and invasion; thus suggesting circTCF2 5 as a new promising marker for bladder cancer [20].

In addition, circRNA-MYLK and VEGFA were significantly up-regulated and co-expressed in bladder cancer; the circRNA-MYLK level was related to its stage and grade progression and it binds directly to miR-29a and relieves suppression for target VEGFA to activate the VEGFA/ VEGFR2 signaling pathway. This accelerates cell proliferation and migration and promotes epithelial-mesenchymal transition [21]. It was also found that circRNA BCRC4 expression was lower in bladder cancer tissues than in adjacent normal tissues. Its forced-expression promotes apoptosis, inhibits viability and increases the miR-101 level which suppresses EZH2 expression. In addition, gambogic acid, a promising natural anti-cancer compound in bladder cancer therapy, increased BCRC4 expression in T24T and UMUC3 cells in a dose-dependent manner [22]. These results indicate that circRNAs function as tumor suppressors or oncogenes in bladder cancer and that they mediate anti-cancer drug function.

Herein, we established that hsa_circ_0000285 expression is lower in cisplatin-resistant bladder cancer patients than in cisplatin-sensitives, and its expression is increased in bladder cancer cell lines. Interestingly, hsa_circ_0000285 expression is significantly down-regulated in RT4/DDP cells compared with parental cells, and combined results indicate that hsa circ_0000285 may be a biomarker in predicting chemotherapy response.

circHIPK3 can regulate cell growth by sponging multiple miRNAs, including miR-193a which is an important mediator of cisplatin-resistance in bladder cancer cells by suppressing AP-2 alpha, homeobox C9, lysyl oxidase-like 4 and the Notch signaling pathway [23-26]. We also observed that while miR-124 is significantly decreased in cancer tissues compared to adjacent tissues, miR-558 is significantly increased (Supplementary Figure 2). MiR-124 and miR-558 are demonstrated circHIPK3 targets, but with opposite roles in bladder cancer: miR-124 functions as tumor suppressor and miR-558 acts as an oncogene [11, 27]. Therefore, the manner in which hsa_circ_0000285 regulates miR-124 and miR-558 expression requires further investigation. These findings provide a possible mechanism for hsa_circ_0000285 in regulating bladder cancer cell proliferation and metastasis; and most likely chemo-sensitivity, but this also requires closer investigation

In conclusion, we established that hsa_circ_0000285 is significantly down-regulated in bladder cancer tissues and plasma and this provides sufficient evidence for hsa circ_0000285 trial as a novel biomarker for bladder cancer, and especially for its role in chemo-sensitivity.
Supplementary information is available in the online version of the paper.

Acknowledgements: This work was supported by the National Natural Science Foundation (grant No. 81041116), Natural Science Foundation of Heilongjiang province (grant no. B2015013), President's innovative and entrepreneurial Foundation of Jiamusi university (grant no. RWSK2017-31) and Foundation of The First Affiliated Hospital of Jiamusi University (grant no. jy2014-009).

\section{References}

[1] RAYN KN, HALE GR, GRAVE GP, AGARWAL PK. New therapies in nonmuscle invasive bladder cancer treatment. Indian J Urol 2018; 34: 11-19. https://doi.org/10.4103/iju. IJU_296_17

[2] VANELLA V, FESTINO L, STRUDEL M, SIMEONE E, GRIMALDI AM et al. PD-L1 inhibitors in the pipeline: Promise and progress. Oncoimmunology 2017; 7: e1365209. https://doi.org/10.1080/2162402X.2017.1365209

[3] MOOSO BA, VINALL RL, MUDRYJ M, YAP SA, DEVERE WHITE WR et al. The role of EGFR family inhibitors in muscle invasive bladder cancer: a review of clinical data and molecular evidence. J Urol 2015; 193: 19-29. https://doi. org/10.1016/j.juro.2014.07.121

[4] GARG M. Epithelial plasticity in urothelial carcinoma: Current advancements and future challenges. World J Stem Cells 2016; 8: 260-267. https://doi.org/10.4252/wjsc.v8.i8.260

[5] CHEN S, ZHU J, WANG F, GUAN Z, GE Y et al. LncRNAs and their role in cancer stem cells. Oncotarget 2017; 8: 110685-110692. https://doi.org/10.18632/oncotarget.22161

[6] HUANG M, ZHONG Z, LV M, SHU J, TIAN Q et al. Comprehensive analysis of differentially expressed profiles of lncRNAs and circRNAs with associated co-expression and ceRNA networks in bladder carcinoma. Oncotarget 2016; 7: 47186-47200. https://doi.org/10.18632/oncotarget.9706

[7] BOLHA L, RAVNIK-GLAVAC M, GLAVAC D. Circular RNAs: Biogenesis, Function, and a Role as Possible Cancer Biomarkers. Int J Genomics 2017; 2017: 6218353. https://doi. org/10.1155/2017/6218353

[8] PATOP IL, KADENER S. circRNAs in Cancer. Curr Opin Genet Dev 2017; 48: 121-127. https://doi.org/10.1016/j. gde.2017.11.007

[9] ZHENG Q, BAO C, GUO W, LI S, CHEN J et al. Circular RNA profiling reveals an abundant circHIPK3 that regulates cell growth by sponging multiple miRNAs. Nat Commun 2016; 7: 11215. https://doi.org/10.1038/ncomms11215

[10] OKHOLM T, NIELSEN MM, HAMILTON MP, CHRISTENSEN LL, VANG $S$ et al. Circular RNA expression is abundant and correlated to aggressiveness in early-stage bladder cancer. NPJ Genom Med 2017; 2: 36. https://doi. org/10.1038/s41525-017-0038-Z

[11] LI Y, ZHENG F, XIAO X, XIE F, TAO D et al. CircHIPK3 sponges miR-558 to suppress heparanase expression in bladder cancer cells. EMBO Rep 2017; 18: 1646-1659. https:// doi.org/10.15252/embr.20164358 
[12] CHEN B, HUANG S. Circular RNA: An emerging noncoding RNA as a regulator and biomarker in cancer. Cancer Lett 2018; 418: 41-50. https://doi.org/10.1016/j.canlet.2018.01.011

[13] Yang F, Liu DY, Guo JT, Ge N, Zhu P et al. Circular RNA circLDLRAD3 as a biomarker in diagnosis of pancreatic cancer. World J Gastroenterol 2017; 23: 8345-8354

[14] SUN H, TANG W, RONG D, JIN H, FU K et al. Hsa circ_0000520, a potential new circular RNA biomarker, is involved in gastric carcinoma. Cancer Biomark 2018; 21: 299-306. https://doi.org/10.3233/CBM-170379

[15] CHEN G, SHI Y, ZHANG Y, SUN J. CircRNA_100782 regulates pancreatic carcinoma proliferation through the IL6STAT3 pathway. Onco Targets Ther 2017; 10: 5783-5794. https://doi.org/10.2147/OTT.S150678

[16] HERMANS T, VOSKUILEN CS, VAN DER HEIJDEN MS, SCHMITZ-DRAGER BJ, KASSOUF W et al. Neoadjuvant treatment for muscle-invasive bladder cancer: The past, the present, and the future. Urol Oncol 2017. https://doi. org/10.1016/j.urolonc.2017.10.014

[17] WEZEL F, VALLO S, ROGHMANN F. Do we have biomarkers to predict response to neoadjuvant and adjuvant chemotherapy and immunotherapy in bladder cancer? Transl Androl Urol 2017; 6: 1067-1080. https://doi.org/10.21037/ tau.2017.09.18

[18] MIAO L, WANG Y, LIN CM, XIONG Y, CHEN N et al. Nanoparticle modulation of the tumor microenvironment enhances therapeutic efficacy of cisplatin. J Control Release 2015; 217: 27-41. https://doi.org/10.1016/j.jconrel.2015.08.027

[19] MARI A, D'ANDREA D, ABUFARAJ M, FOERSTER B, KIMURA $S$ et al. Genetic determinants for chemo- and radiotherapy resistance in bladder cancer. Transl Androl Urol 2017; 6: 1081-1089. https://doi.org/10.21037/ tau.2017.08.19
[20] ZHONG Z, LV M, CHEN J. Screening differential circular RNA expression profiles reveals the regulatory role of circTCF25-miR-103a-3p/miR-107-CDK6 pathway in bladder carcinoma. Sci Rep 2016; 6: 30919. https://doi. org/10.1038/srep30919

[21] ZHONG Z, HUANG M, LV M, HE Y, DUAN C et al. Circular RNA MYLK as a competing endogenous RNA promotes bladder cancer progression through modulating VEGFA/ VEGFR2 signaling pathway. Cancer Lett 2017; 403: 305-317. https://doi.org/10.1016/j.canlet.2017.06.027

[22] LI B, XIE F, ZHENG FX, JIANG GS, ZENG FQ et al. Overexpression of CircRNA BCRC4 regulates cell apoptosis and MicroRNA-101/EZH2 signaling in bladder cancer. J Huazhong Univ Sci Technolog Med Sci 2017; 37: 886-890. https://doi.org/10.1007/s11596-017-1822-9

[23] Zhou J, Duan H, Xie Y, Ning Y, Zhang X et al. MiR-193a-5p Targets the Coding Region of AP-2alpha mRNA and Induces Cisplatin Resistance in Bladder Cancers. J Cancer 2016; 7: 1740-1746. https://doi.org/10.7150/jca.15620

[24] Lv L, Li Y, Deng H, Zhang C, Pu Y et al. MiR-193a-3p promotes the multi-chemoresistance of bladder cancer by targeting the HOXC9 gene. Cancer Lett 2015; 357: 105-113. https://doi.org/10.1016/j.canlet.2014.11.002

[25] DENG H, LV L, LI Y, ZHANG C, MENG F et al. miR-193a-3p regulates the multi-drug resistance of bladder cancer by targeting the LOXL4 gene and the oxidative stress pathway. Mol Cancer 2014; 13: 234. https://doi.org/10.1186/1476-4598-13-234

[26] LV L, DENG H, LI Y, ZHANG C, LIU X et al. The DNA methylation-regulated miR-193a-3p dictates the multichemoresistance of bladder cancer via repression of SRSF2/ PLAU/HIC2 expression. Cell Death Dis 2014; 5: e1402. https://doi.org/10.1038/cddis.2014.367

[27] WANG X, WU Q, XU B, WANG P, FAN W et al. MiR-124 exerts tumor suppressive functions on the cell proliferation, motility and angiogenesis of bladder cancer by finetuning UHRF1. FEBS J 2015; 282: 4376-4388. https://doi. org/10.1111/febs.13502 